\title{
ANALYTICITY OF DIMENSIONS FOR HYPERBOLIC SURFACE DIFFEOMORPHISMS
}

\author{
M. POLLICOTT
}

(Communicated by Nimish Shah)

\begin{abstract}
In this note we give a simple proof that the Hausdorff dimension of the basic set for a real analytic Smale horseshoe map depends analytically on the transformation. This method is based on the use of dynamical zeta functions. We prove analogous statements for the value of the pointwise dimension of the measure of maximal entropy and then use this to address an interesting question raised by Damanik and Gorodetski.
\end{abstract}

\section{INTRODUCTION}

The analytic dependence of the Hausdorff dimension of dynamically defined sets on the defining maps has been studied in a number of settings; for example, Ruelle's work on hyperbolic Julia sets [15, which in turn was inspired by Bowen's work on limits sets for quasi-Fuchsian groups [2]. In this note we describe analogous results for Axiom A diffeomorphisms on surfaces.

Let $\Lambda$ be a basic set for a real analytic Axiom A diffeomorphism $f: M \rightarrow M$ on a compact surface $M$. We can associate to the set its Hausdorff $\operatorname{dimension} \operatorname{dim}_{H}\left(\Lambda_{f}\right)$. Let us consider a one parameter family of real analytic Axiom A diffeomorphisms $f_{\lambda} \in C^{\omega}(M), \lambda \in(-\epsilon, \epsilon)$, and let $\Lambda_{f_{\lambda}}$ denote the basic sets associated by structural stability.

Theorem 1. The Hausdorff dimension $\operatorname{dim}_{H}\left(\Lambda_{f_{\lambda}}\right)$ of the basic set $\Lambda_{f_{\lambda}}$ for a $C^{\omega}$ surface diffeomorphism depends analytically on $\lambda \in(-\epsilon, \epsilon)$.

The dependence was shown to be continuous by McCluskey and Manning [10. and $C^{\infty}$ by a result of Mañé [9] (cf. also [7]). The approach in [9] was to first prove the smooth dependence of the structural stability map and the associated potential, and then deduce from this the results on dimension. A similar approach was used in [6] to show both the $C^{\infty}$ and $C^{\omega}$ dependence of the topological entropy for Anosov flows. We do not know a suitable reference in the analytic case, where an extra complication is that one would have to deal with infinite dimensional Banach manifolds and their complexifications. There are related results stated for complex Hénon attractors in [16] and [17, which both rely on an extension of Mañé's Banach space approach to the analytic function (but without providing details; cf. p. 860 of [16]). However, we bypass this difficulty entirely by adopting a different "finite dimensional approach" using individual closed orbits, which works particularly well in establishing analyticity and is similar in spirit to both Ruelle's original approach for real repellers in [15] and the approach in [6].

Received by the editors May 10, 2013 and, in revised form, November 30, 2013.

2010 Mathematics Subject Classification. Primary 37D35, 37F35. 
We can also consider a related problem. We can associate to the measure of maximal entropy $\mu_{f}$ its Hausdorff measure $\operatorname{dim}_{H}\left(\mu_{f}\right)$, i.e., the infimum of the Hausdorff dimensions of sets of full measure.

Theorem 2. The Hausdorff dimension $\operatorname{dim}_{H}\left(\mu_{\lambda}\right)$ of the maximal measure $\mu_{f_{\lambda}}$ depends analytically on $\lambda \in(-\epsilon, \epsilon)$.

Even in the case that $\Lambda$ is the entire surface, Theorem 2 has non-empty content. In particular, we have the following corollary, which was conjectured by DamanikGorodetski ([3, §1, Remark (i)]).

Corollary 3. The density of states for the weakly coupled Fibonacci Hamiltonian depends analytically on the potential.

In section 2 we give some basic definitions and preliminary results on Hausdorff dimension. In section 3 we introduce a complex function, called the differential zeta function, and some properties of its meromorphic extension. Then in section 4 we show how to use these results to prove Theorem 1

In section 5 we give some basic definitions and preliminary results for pointwise dimension. In section 6 we introduce two new complex functions, a zeta function and an eta function, and some properties of their meromorphic extensions. Then, in section 7 we use these results to prove Theorem 2 .

In section 8 we recall how Corollary 3 follows from Theorem 2

In Appendix 9 we sketch the proof of Lemma 16 from section 6.

\section{BASIC SETS AND THEIR HAUSDORFF DIMENSION}

We begin by recalling some classical results on Axiom A diffeomorphisms [1]. Let $f: M \rightarrow M$ be a real analytic diffeomorphism of a compact manifold $M$.

Definition 4. We call an $f$-invariant closed set $\Lambda \subset M$ a basic set if:

(1) $f: \Lambda \rightarrow \Lambda$ is hyperbolic, i.e., there exists a $D f$-invariant splitting $T M=$ $E^{+} \oplus E^{-}$and constants $C>0,0<\lambda<1$ such that

(a) $\left\|D f^{n} \mid E^{+}\right\| \leq C \lambda^{n}$ for $n \geq 0$; and

(b) $\left\|D f^{-n} \mid E^{-}\right\| \leq C \lambda^{n}$ for $n \geq 0$;

(2) there exists an open neighbourhood $U \supset \Lambda$ such that $\Lambda=\bigcap_{n \in \mathbb{Z}} f^{-n} U$;

(3) $f: \Lambda \rightarrow \Lambda$ is transitive; and

(4) the periodic orbits are dense in $f: \Lambda \rightarrow \Lambda$.

The hyperbolicity is reflected in stable and unstable manifolds of Axiom A basic sets.

We next recall the definition of the Hausdorff dimension.

Definition 5. Given a set $X, d>0$ and $\epsilon>0$ we define

$$
H_{\epsilon}^{d}(X)=\inf _{\mathcal{U}}\left\{\sum_{i} \operatorname{diam}\left(U_{i}\right)^{d}\right\}
$$

where the infimum is over all open covers $\mathcal{U}=\left\{U_{i}\right\}$ whose elements have diameter at most $\epsilon>0$. The Hausdorff dimension is given by

$$
\operatorname{dim}_{H}(X)=\inf \left\{d \geq 0: \lim _{\epsilon \rightarrow 0} H_{\epsilon}^{d}(X)=0\right\} .
$$

Let $\delta_{f}=\operatorname{dim}_{H}\left(\Lambda_{f}\right)$ denote the Hausdorff dimension of the limit set $\Lambda$. 
Definition 6. Given $\epsilon>0$ we can associate to each point $x \in \Lambda$ the (local) stable and unstable manifolds $W_{\epsilon}^{+}(x)=\left\{y: d\left(f^{n} x, f^{n} y\right) \leq \epsilon, \forall n \geq 0\right\}$ and $W_{\epsilon}^{-}(x)=$ $\left\{y: d\left(f^{-n} x, f^{-n} y\right) \leq \epsilon, \forall n \geq 0\right\}$.

In particular, $f\left(W_{\epsilon}^{+}(x)\right) \subset W_{\epsilon}^{+}(f(x))$ and $f^{-1}\left(W_{\epsilon}^{-}(x)\right) \subset W_{\epsilon}^{-}\left(f^{-1}(x)\right)$.

Let $\delta_{f}^{+}=\operatorname{dim}_{H}\left(\Lambda_{f} \cap W_{\epsilon}^{+}\right)(x)$ and $\delta_{f}^{-}=\operatorname{dim}_{H}\left(\Lambda_{f} \cap W_{\epsilon}^{-}(x)\right)$ be the Hausdorff dimension of the intersection of $\Lambda_{f}$ with the local stable and unstable manifolds, respectively.

Lemma 7 (McCluskey-Manning). $\delta_{f}^{+}$and $\delta_{f}^{-}$are independent of $x \in \Lambda_{f}$ and we can write $\delta_{f}=\delta_{f}^{+}+\delta_{f}^{-}$.

This can be deduced from Theorem 1 and Theorem 2 in [10].

\section{ZETA FUNCTIONS}

There is a useful characterization of the dimensions $\delta^{+}, \delta^{-}$in terms of the differential zeta functions [1]. Let $\operatorname{Fix}\left(f^{n}\right)=\left\{x \in \Lambda: f^{n} x=x\right\}$ be the set of fixed points for $f^{n}$.

Definition 8. For any periodic point $f^{n}(x)=x \in \Lambda$, we can associate the two weights

$$
\lambda_{f}^{-}(x)=-\log \left|D_{x} f^{n}\right| E_{x}^{-} \mid \quad \text { and } \quad \lambda_{f}^{+}(x)=-\log \left|D_{x} f^{n}\right| E_{x}^{+} \mid .
$$

We can then define the stable differential zeta functions and unstable differential zeta functions, respectively, by

$$
\zeta_{f}^{+}(s)=\exp \left(\sum_{n=1}^{\infty} \frac{1}{n} \sum_{x \in \operatorname{Fix}\left(f^{n}\right)} e^{-s \lambda_{f}^{+}(x)}\right)
$$

and

$$
\zeta_{f}^{-}(s)=\exp \left(\sum_{n=1}^{\infty} \frac{1}{n} \sum_{x \in \operatorname{Fix}\left(f^{n}\right)} e^{-s \lambda_{f}^{-}(x)}\right) .
$$

These converge for $\operatorname{Re}(s)$ sufficiently large.

We can define Lipschitz functions $\phi^{+}: \Lambda \rightarrow \mathbb{R}$ and $\phi^{-}: \Lambda \rightarrow \mathbb{R}$ by

$$
\phi^{+}(x)=-\log \left|D_{x} f\right| E_{x}^{+} \mid \text {and } \phi^{-}(x)=-\log \left|D_{x} f\right| E_{x}^{-} \mid,
$$

respectively. The Lipschitz regularity of the functions is a consequence of the Lipschitz regularity of the stable and unstable laminations.

We denote for each $t \in \mathbb{R}$,

$$
\begin{aligned}
P\left(-t \phi^{+}(x)\right) & :=\limsup _{n \rightarrow+\infty} \frac{1}{n} \log \sum_{x \in \operatorname{Fix}\left(f^{n}\right)} \exp \left(-t \sum_{k=0}^{n-1} \phi^{+}\left(f^{k} x\right)\right) \\
& =\limsup _{n \rightarrow+\infty} \frac{1}{n} \log \sum_{x \in \operatorname{Fix}\left(f^{n}\right)} e^{-t \lambda^{+}(x)}
\end{aligned}
$$


and

$$
\begin{aligned}
P\left(-t \phi^{-}(x)\right) & :=\limsup _{n \rightarrow+\infty} \frac{1}{n} \log \sum_{x \in \operatorname{Fix}\left(f^{n}\right)} \exp \left(-t \sum_{k=0}^{n-1} \phi^{-}\left(f^{k} x\right)\right) \\
& =\limsup _{n \rightarrow+\infty} \frac{1}{n} \log \sum_{x \in \operatorname{Fix}\left(f^{n}\right)} e^{-t \lambda^{-}(x)} .
\end{aligned}
$$

A lot is known about the domain of these zeta functions, but for our purposes we only require the following:

Lemma 9. The differential zeta functions satisfy the following properties:

(1) $\zeta_{f}^{+}(s)$ converges to an analytic function on the half-plane $\operatorname{Re}(s)>\delta_{f}^{+}$and has a meromorphic extension to a neighbourhood of a simple pole at $s=\delta_{f}^{+}$; and

(2) $\zeta_{f}^{-}(s)$ converges to an analytic function on the half-plane $\operatorname{Re}(s)>\delta_{f}^{-}$and has a meromorphic extension to a neighbourhood of a simple pole at $s=\delta_{f}^{-}$.

Proof. It is immediate from the definitions that: $\zeta_{f}^{+}(s)$ converges to an analytic function provided $P\left(-\operatorname{Re}(s) \phi^{+}(x)\right)<0$ and diverges provided $P\left(-\operatorname{Re}(s) \phi^{+}(x)\right)>$ 0 ; and $\zeta_{f}^{-}(s)$ converges to an analytic function provided $P\left(-\operatorname{Re}(s) \phi^{-}(x)\right)<0$; and diverges provided $P\left(-\operatorname{Re}(s) \phi^{-}(x)\right)>0$. Furthermore, by [10], $t \mapsto P\left(-t \phi_{f}^{+}\right)$, $P\left(-t \phi_{f}^{-}\right)$are strictly decreasing and $P\left(-\delta^{-} \phi^{-}\right)=0$ and $P\left(\delta^{+} \phi^{+}\right)=0$, giving the half-planes of convergence.

The meromorphic extensions to $\zeta_{f}^{-}(s)$ and $\zeta_{f}^{+}(s)$ to neighbourhoods of $\delta^{-}$and $\delta^{+}$, respectively, follows from a result of Ruelle (Theorem 1 of [14, and $\S 5.20$ of [13]) (cf. also [12, §5]).

\section{Proof of Theorem 1}

Consider the family of analytic functions $f_{\lambda},-\epsilon<\lambda<\epsilon$. A key to the proof of the theorems in our approach is the following simple lemma.

Lemma 10. There exist neighbourhoods $(-\epsilon, \epsilon) \subset U \subset \mathbb{C}$ and $\delta^{-} \in V \subset \mathbb{C}$ such that $U \times V \ni(\lambda, s) \mapsto \zeta_{f_{\lambda}}^{-}(s)^{-1}$ is bianalytic. There is a corresponding result for $\zeta_{f_{\lambda}}^{+}(s)^{-1}$.

Proof. By the basic structural stability theorem for Axiom A diffeomorphisms there is a natural bijection between the periodic points of $f=f_{0}$ and $f=f_{\lambda}$. We write $f_{\lambda}^{n}\left(x_{\lambda}\right)=x_{\lambda}$ for the associated periodic point and then it is easy to see that $(-\epsilon, \epsilon) \ni$ $\lambda \rightarrow x_{\lambda} \in M$ is analytic and, moreover, there exists a common neighbourhood $U \supset(-\epsilon, \epsilon)$ for the complexification $U \ni \lambda \rightarrow x_{\lambda}$, where $U \subset \mathbb{C}$ works for any periodic point (cf. [6, Proposition 1.1]). Thus in the region where the zeta function $\zeta_{f_{\lambda}}^{-}(s)$ converges to non-zero analytic function we see that $(\lambda, s) \mapsto \zeta_{f_{\lambda}}^{-}(s)^{-1}$ is bianalytic. Thus using Hartog's theorem [8] we have that $(\lambda, s) \mapsto \zeta_{f_{\lambda}}^{-}(s)^{-1}$ is bianalytic

Let us assume without loss of generality that $\Gamma \subset V$ is a simple closed curve inside which $\zeta_{f_{\lambda}}^{-}(s)^{-1}$ contains the single zero $\delta_{f_{\lambda}}^{-}$. We can then use Cauchy's theorem to write

$$
\delta_{f_{\lambda}}^{-}=\frac{1}{2 \pi i} \int_{\Gamma} \frac{\zeta_{f_{\lambda}}^{-}(\xi)}{\zeta_{f_{\lambda}}^{-}(\xi)} \xi d \xi,
$$


which, when combined with Lemma 10, allows us to deduce that $U \ni \lambda \mapsto \delta_{f_{\lambda}}^{-}$is analytic. A similar argument involving $\zeta_{f_{\lambda}}^{+}(s)$ applies to show that $\lambda \mapsto \delta_{f_{\lambda}}^{+}$.

The proof of Theorem 1 is now a simple consequence of Lemma 7 since

$$
U \ni \lambda \mapsto \operatorname{dim}_{H}\left(\Lambda_{f_{\lambda}}\right)=: \delta_{f}=\delta_{f_{\lambda}}^{+}+\delta_{f_{\lambda}}^{-}
$$

is analytic, as required.

\section{Pointwise Dimension}

We recall that for a given $f$-invariant measure $\mu$ that the pointwise dimension at $x$ is defined by

$$
d_{\mu}(x)=\lim _{\epsilon \rightarrow 0} \frac{\log \mu(B(x, \epsilon))}{\log \epsilon},
$$

when the limit converges.

The stable and unstable manifolds provide a local product structure, i.e., for sufficiently small $\epsilon>0$ there exists $\delta>0$ such that for each $x \in \Lambda$ the map

$$
[\cdot, \cdot]_{x}:\left(W_{\epsilon}^{+}(x) \cap \Lambda\right) \times\left(W_{\epsilon}^{-}(x) \cap \Lambda\right) \rightarrow \Lambda
$$

defined by

$$
\left[y, y^{\prime}\right]_{x}=W_{\epsilon}^{-}(y) \cap W_{\epsilon}^{+}\left(y^{\prime}\right)
$$

is a local homeomorphism onto its image.

Lemma 11. In the particular case of the measure of maximal entropy, we can locally write the measure as a product $\mu^{+} \times \mu^{-}$.

We can then write

$$
d_{\mu}^{-}(x)=\lim _{\epsilon \rightarrow 0} \frac{\log \mu^{-}\left(B^{-}(x, \epsilon)\right)}{\log \epsilon},
$$

where $B^{-}(x, \epsilon) \subset W^{-}(x, \epsilon)$ is an $\epsilon$-ball in the unstable manifold, and

$$
d_{\mu}^{+}(x)=\lim _{\epsilon \rightarrow 0} \frac{\log \mu^{+}\left(B^{-}(x, \epsilon)\right)}{\log \epsilon},
$$

where $B^{+}(x, \epsilon) \subset W^{+}(x, \epsilon)$ is an $\epsilon$-ball in the unstable manifold.

A useful characterization of $d_{\mu}^{+}(x)$ and $d_{\mu}^{-}(x)$ is given by the following lemma [18].

Lemma 12 (L.-S. Young). For any Axiom A diffeomorphism:

(1) The functions $d_{\mu}^{+}(x)$ and $d_{\mu}^{-}(x)$ are constant a.e. $(\mu)$.

(2) We have that $d_{\mu}(x)=d_{\mu}^{+}(x)+d_{\mu}^{-}(x)$.

(3) The pointwise dimensions given by

$$
d_{\mu}^{-}(x)=\frac{h(f)}{\int \phi^{-}(x) d \mu(x)} \quad \text { and } \quad d_{\mu}^{+}(x)=\frac{h(f)}{\int \phi^{+}(x) d \mu(x)} .
$$

Here the topological entropy $h(f)$ is constant under perturbation and so the remaining problem is to understand the dependence of the Lyapunov exponent. 


\section{Generalized Zeta Functions}

We can define the following generalizations of the differential zeta functions.

Definition 13. The generalized stable differential zeta functions and unstable differential zeta functions are defined, respectively, by

$$
\zeta_{f}^{+}(z, s)=\exp \left(\sum_{n=1}^{\infty} \frac{z^{n}}{n} \sum_{f^{n} x=x} e^{-s \lambda_{f}^{+}(x)}\right)
$$

and

$$
\zeta_{f}^{-}(z, s)=\exp \left(\sum_{n=1}^{\infty} \frac{z^{n}}{n} \sum_{f^{n} x=x} e^{-s \lambda_{f}^{-}(x)}\right)
$$

which converge to a non-zero analytic function for $\operatorname{Re}(s)$ sufficiently large and $|z|$ sufficiently small.

The generalization of Lemma 9 is the following.

Lemma 14 (after Ruelle). The zeta functions have the following properties:

(1) The zeta function $\zeta_{f}^{-}(z, s)$ converges to a non-zero analytic function on the domain $|z|<\exp \left(P\left(-R e(s) \phi^{-}\right)\right)$which has a meromorphic extension to a neighbourhood of $\left(e^{-h(f)}, 0\right)$. More precisely,

$$
\zeta_{f}^{-}(z, s)\left(1-z e^{P\left(-s \phi^{-}\right)}\right)
$$

is analytic in a neighbourhood of $\left(e^{-h(f)}, 0\right)$.

(2) The zeta function $\zeta_{f}^{+}(z, s)$ converges to a non-zero analytic function on the domain $|z|<\exp \left(P\left(-R e(s) \phi^{+}\right)\right)$which has a meromorphic extension to a neighbourhood of $\left(e^{-h(f)}, 0\right)$. More precisely,

$$
\zeta_{f}^{+}(z, s)\left(1-z e^{P\left(-s \phi^{+}\right)}\right)
$$

is analytic in a neighbourhood of $\left(e^{-h(f)}, 0\right)$.

This is again a consequence of a more general result of Ruelle ([13, §5.29]); cf. also [12, Theorem 5.6].

We need to modify the complex functions to take a form which allows the Lyapunov exponents to be read off more easily.

Definition 15. We define:

$$
\eta_{f}^{-}(z)=-\left.\frac{\partial}{\partial s} \log \zeta_{f}^{-}(z, s)\right|_{s=0}=\sum_{n=1}^{\infty} \frac{z^{n}}{n} \sum_{f^{n} x=x} \lambda_{f}^{-}(x)
$$

and

$$
\eta_{f}^{+}(z)=-\left.\frac{\partial}{\partial s} \log \zeta_{f}^{+}(z, s)\right|_{s=0}=\sum_{n=1}^{\infty} \frac{z^{n}}{n} \sum_{f^{n} x=x} \lambda_{f}^{+}(x)
$$

which both converge for $|z|<e^{-h(f)}$.

This brings us to the following fairly routine result. 
Lemma 16. Let $\mu_{f}$ is the measure of maximal entropy for $f$ (i.e., $h\left(\mu_{f}\right)=h(f)$ ).

(1) The function $\eta_{f}^{-}(z)$ is non-zero and analytic for $|z|<e^{-h(f)}$ and has a meromorphic extension to a neighbourhood of a simple pole at $z=e^{-h(f)}$ with residue $e^{-h(f)} \int \phi^{-} d \mu_{f}$.

(2) The function $\eta_{f}^{+}(z)$ is non-zero and analytic for $|z|<e^{-h(f)}$ and has a meromorphic extension to a neighbourhood of a simple pole at $z=e^{-h(f)}$ with residue $e^{-h(f)} \int \phi^{+} d \mu_{f}$.

This follows easily from the results in [13] and [12, but we sketch the details in the appendix for the reader's convenience.

\section{Proof of Theorem 2}

Consider the family of analytic functions $f_{\lambda}, \lambda \in(-\epsilon, \epsilon)$. A key to the proof of the theorems in our approach is the following simple lemma.

Lemma 17. There exist neighbourhoods $(-\epsilon, \epsilon) \subset U \subset \mathbb{C}$ and $e^{-h(f)} \in V \subset \mathbb{C}$ such that $U \times V \ni(\lambda, s) \mapsto \eta_{f_{\lambda}}^{-}(z)^{-1}$ is bianalytic. There is a corresponding result for $\eta_{f_{\lambda}}^{+}(z)^{-1}$.

Proof. The proof is analogous to that of Lemma 10. By the basic structural stability theorem for Axiom A diffeomorphisms there is a natural bijection between the periodic points of $f=f_{0}$ and $f=f_{\lambda}$. We write $f_{\lambda}^{n}\left(x_{\lambda}\right)=x_{\lambda}$ for the associated periodic point and then it is easy to see that $(-\epsilon, \epsilon) \ni \lambda \rightarrow x_{\lambda} \in M$ is analytic and, moreover, there exists a common neighbourhood $U \supset(-\epsilon, \epsilon)$ for the complexification $U \ni \lambda \rightarrow x_{\lambda}$, where $U \subset \mathbb{C}$ works for any periodic point. Thus in the region where the eta function $\eta_{f_{\lambda}}^{-}(z)$ converges to non-zero analytic function we see that $(\lambda, z) \mapsto \eta_{f_{\lambda}}^{-}(z)^{-1}$ is bianalytic. Thus using Hartog's theorem [8] we have that $(\lambda, z) \mapsto \eta_{f_{\lambda}}^{-}(z)^{-1}$ is bianalytic

Let us assume without loss of generality that $\Gamma \subset V$ is a simple closed curve inside which $\eta_{f_{\lambda}}^{-}(z)^{-1}$ contains the single zero $e^{-h(f)}$. We can then use Cauchy's theorem to write the residue:

$$
e^{-h(f)} \int \phi^{-} d \mu_{f_{\lambda}}=\frac{1}{2 \pi i} \int_{\Gamma} \eta_{f_{\lambda}}^{-}(\xi) d \xi
$$

which, when combined with Lemma 17, allows us to deduce that $U \ni \lambda \mapsto \int \phi^{-} d \mu_{f_{\lambda}}$ is analytic. A similar argument involving $\eta_{f_{\lambda}}^{+}(z)$ applies to show that $U \ni \lambda \mapsto$ $\int \phi^{+} d \mu_{f_{\lambda}}$ is analytic.

The proof of Theorem 2 is now a simple consequence of Lemma 12 since

$$
U \ni \lambda \mapsto d_{\mu_{f}}=d_{\mu_{f}}^{+}+d_{\mu_{f}}^{-}=\frac{h(f)}{\int \phi^{-} d \mu_{f_{\lambda}}}+\frac{h(f)}{\int \phi^{+} d \mu_{f_{\lambda}}}
$$

is analytic, as required.

\section{Application to the density of states: Proof of Corollary 3}

We can consider the Fibonacci Hamiltonian:

$$
\left(H_{V, \omega} \psi\right)(n)=\psi(n+1)+\psi(n-1)+V \chi_{[1-\alpha, 1)}(\{n \alpha+\omega\}) \psi(n)
$$

for $\psi \in l^{2}(\mathbb{Z})$, where $0 \leq \omega<1, \alpha$ is the Golden Mean and $V>0$, sufficiently small, is a parameterization of the family. The spectrum is independent of the choice of 
$\omega$, by unique ergodicity of irrational rotations on the circle and we denote it by $\operatorname{Spect}\left(H_{V}\right)$. Since the operator is self-adjoint the $\operatorname{spectrum} \operatorname{Spect}\left(H_{V}\right)$ is contained in the real line and, finally, the spectrum is a Cantor set of zero Lebesgue measure.

By the spectral theorem, there is a spectral measure $\mu_{V, \omega}$ supported on Spect $\left(H_{V}\right) \subset \mathbb{R}$ such that for any continuous compactly supported continuous function $g: \mathbb{R} \rightarrow \mathbb{R}$ we have $\left\langle\delta_{0}, g\left(H_{v}\right) \delta_{0}\right\rangle=\int g(t) d \mu_{V, \omega}(t)$. The density of states measure $\mu_{V}$ on $\operatorname{Spect}\left(H_{V, \omega}\right) \subset \mathbb{R}$ is then given by integrating with respect to $\omega$, i.e., for any continuous compactly supported continuous function $g: \mathbb{R} \rightarrow \mathbb{R}$ we have

$$
\int_{0}^{1}\left\langle\delta_{0}, g\left(H_{V}\right) \delta_{0}\right\rangle d \omega=\int g(t) d \mu_{V}(t) .
$$

We can associate to $\mu_{V}$ its pointwise dimension, i.e., for a.e. $\left(\mu_{V}\right) x \in \operatorname{Spect}\left(H_{V, \omega}\right)$ we have

exists, and is constant.

$$
d_{\mu_{V}}(x):=\lim _{\epsilon \rightarrow 0} \frac{\log \mu_{V}(B(x, \epsilon))}{\log \epsilon}
$$

In [3] an interesting connection is made between the measure $\mu_{V}$ and the pointwise dimension of the measure of maximal entropy for a horseshoe. To define the corresponding hyperbolic map, we can consider the trace map $T: \mathbb{R}^{3} \rightarrow \mathbb{R}^{3}$ defined by

$$
T(x, y, z)=(2 x y-z, x, y) .
$$

For each $V$, this map preserves the surface

$$
S_{V}=\left\{(x, y, z) \in \mathbb{R}^{3}: x^{2}+y^{2}+z^{2}-2 x y z=1+\frac{V^{2}}{4}\right\}
$$

and we will consider the restrictions $T \mid S_{V}$, and let $\Omega_{V} \subset S_{V}$ be the non-wandering set of this map. For $V>0$ sufficiently small the set $\Omega_{V}$ is a basic set of an Axiom A diffeomorphism which depends analyticially on $V$. The Hausdorff dimension of the spectrum coincides with the Hausdorff dimension if the intersection of $\Omega_{V}$ with local unstable manifolds and depends analytically (see [4, Theorem 6.5 and its proof]; cf. [5]).

As before, we can consider the measure of maximal entropy $\mu$ for $T: S_{V} \rightarrow S_{V}$ and the pointwise dimension $d_{\mu^{-}}$of its projection along the stable direction.

The following nice result relates the value of the pointwise dimension of the density of states measure $\mu_{V}$ to the value of the pointwise dimension of the maximal measure $d_{\mu}^{-}$on unstable manifolds $[3$.

Proposition 18 (Damanik-Gorodetski). $d_{\mu_{V}}=d_{\mu}^{-}$.

Damanik and Gorodetski observed that the value of $d_{\mu_{V}}$ depends smoothly on $V$. We have as a corollary of our theorem that the value of $d_{\mu_{V}}$ depends analytically on $V$.

Remark 19 (Multifractal analysis). Given $0<V_{0}<V<V_{1}$, for each $t$ we can associate

$$
\mathcal{F}(\alpha, V)=\operatorname{dim}_{H}\left\{x: d_{\mu_{V}}(x)=t\right\} .
$$

Multifractal analysis gives that we can choose $0<a<b$ such that

$$
\begin{aligned}
(a, b) \times\left(V_{0}, V_{1}\right) & \rightarrow \mathbb{R} \\
(t, V) & \mapsto \mathcal{F}(t, V)
\end{aligned}
$$

is analytic. 


\section{Appendix: Proof of Lemma 16}

This is a simple exercise using symbolic dynamics (and we refer the reader to [12 for more details). We can model the diffeormorphism by a subshift of finite type $\sigma: X_{A} \rightarrow X_{A}$ and consider a Hölder function $r: X_{A} \rightarrow \mathbb{R}$ in place of either $\phi^{-}$and then we can consider

$$
\widetilde{\zeta}^{-}(z, s)=\exp \left(\sum_{n=1}^{\infty} \frac{z^{n}}{n} \sum_{\sigma^{n} x=x} e^{-s r^{n}(x)}\right) .
$$

In particular, we can write $\widetilde{\zeta}^{-}(z, s)=\psi(z, s) \zeta^{-}(z, s)$ where $\psi(z, s)$ is analytic in a neighbourhood of $\left(e^{-h(f)}, 0\right)$. We can then write

$$
\begin{aligned}
\widetilde{\eta}_{f}^{-}(z) & :=-\left.\frac{\partial}{\partial s} \log \widetilde{\zeta}^{-}(z, s)\right|_{s=0} \\
& =\eta_{f}^{-}(z)-\left.\frac{\partial}{\partial s} \psi(z, s)\right|_{s=0} / \psi(z, 0)
\end{aligned}
$$

where $\widetilde{\eta}_{f}^{-}(z)$ and $\eta_{f}^{-}(z)$ have the same residue at the simple pole $z=e^{-h(f)}$ (and where we observe $h(f)=h(\sigma))$. Observe that $\widetilde{\eta}_{f}^{-}(z)$ has a pole at $z=e^{-h(f)}$ since

$$
\widetilde{\zeta}^{-}(z, s)=\frac{\rho(z, s)}{1-z e^{P(-s r)}},
$$

where $\rho(z, s)$ is analytic and non-zero in a neighbourhood of $\left(e^{-h(f)}, 0\right)$; cf. [12]. Thus taking the logarithmic derivative in $s$ gives:

$$
\frac{\left(\frac{\partial P(-s r)}{\partial s}\right) z e^{P(-s r)}}{1-z e^{P(-s r)}}+\frac{\frac{\partial \rho}{\partial s}(z, s)}{\rho(z, s)}
$$

and then setting $s=0$ gives that the residue for $\widetilde{\eta}_{f}^{-}(z)$ (and thus $\eta_{f}^{-}(z)$ ) at $z=$ $e^{-h(f)}$ is

$$
\begin{aligned}
& \lim _{z \rightarrow e^{-h(f)}}\left(z-e^{-h(f)}\right) \frac{\left(\left.\frac{\partial P(-s r)}{\partial s}\right|_{s=0}\right)}{1-z e^{h(f)}} \\
= & e^{-h(f)} \lim _{z \rightarrow e^{-h(f)}}\left(\frac{z-e^{-h(f)}}{e^{-h(f)}-z}\right)\left(\frac{\partial P(-s r)}{\partial s}\right) \\
= & e^{-h(f)} \int \phi^{-} d \mu_{f}
\end{aligned}
$$

since

(1) $e^{P(-s r)} \rightarrow e^{h(f)}$ as $s \rightarrow 0$;

(2) $\left.\frac{\partial P(-s r)}{\partial s}\right|_{s=0}=-\int \phi^{-} d \mu_{f}$

(cf. [12, Proposition 4.10]). The identity for the residue for $\widetilde{\eta}_{f}^{+}(z)$ (and thus $\eta_{f}^{+}(z)$ ) is proved analogously.

\section{REFERENCES}

[1] Rufus Bowen, Equilibrium states and the ergodic theory of Anosov diffeomorphisms, Lecture Notes in Mathematics, Vol. 470, Springer-Verlag, Berlin-New York, 1975. MR0442989 (56 \#1364)

[2] Rufus Bowen, Hausdorff dimension of quasicircles, Inst. Hautes Études Sci. Publ. Math. 50 (1979), 11-25. MR556580 (81g:57023) 
[3] David Damanik and Anton Gorodetski, The density of states measure of the weakly coupled Fibonacci Hamiltonian, Geom. Funct. Anal. 22 (2012), no. 4, 976-989, DOI 10.1007/s00039012-0173-8. MR 2984122

[4] Serge Cantat, Bers and Hénon, Painlevé and Schrödinger, Duke Math. J. 149 (2009), no. 3, 411-460, DOI 10.1215/00127094-2009-042. MR2553877 (2011f:37077)

[5] Martin Casdagli, Symbolic dynamics for the renormalization map of a quasiperiodic Schrödinger equation, Comm. Math. Phys. 107 (1986), no. 2, 295-318. MR863644 (88d:58101)

[6] A. Katok, G. Knieper, M. Pollicott, and H. Weiss, Differentiability and analyticity of topological entropy for Anosov and geodesic flows, Invent. Math. 98 (1989), no. 3, 581-597, DOI 10.1007/BF01393838. MR 1022308 (90i:58150)

[7] Miaohua Jiang and Rafael de la Llave, Smooth dependence of thermodynamic limits of SRBmeasures, Comm. Math. Phys. 211 (2000), no. 2, 303-333, DOI 10.1007/s002200050814. MR:1754517 (2001g:37038)

[8] Steven G. Krantz, Function theory of several complex variables, 2nd ed., The Wadsworth \& Brooks/Cole Mathematics Series, Wadsworth \& Brooks/Cole Advanced Books \& Software, Pacific Grove, CA, 1992. MR1162310 (93c:32001)

[9] Ricardo Mañé, The Hausdorff dimension of horseshoes of diffeomorphisms of surfaces, Bol. Soc. Brasil. Mat. (N.S.) 20 (1990), no. 2, 1-24, DOI 10.1007/BF02585431. MR.1143171 (92k:58200)

[10] Heather McCluskey and Anthony Manning, Hausdorff dimension for horseshoes, Ergodic Theory Dynam. Systems 3 (1983), no. 2, 251-260, DOI 10.1017/S0143385700001966. MR742227 (85j:58127)

[11] William Parry, Synchronisation of canonical measures for hyperbolic attractors, Comm. Math. Phys. 106 (1986), no. 2, 267-275. MR855312 (88b:58088)

[12] William Parry and Mark Pollicott, Zeta functions and the periodic orbit structure of hyperbolic dynamics (English, with French summary), Astérisque 187-188 (1990), 268. MR.1085356 (92f:58141)

[13] David Ruelle, Thermodynamic formalism, Encyclopedia of Mathematics and its Applications, vol. 5, Addison-Wesley Publishing Co., Reading, Mass., 1978. The mathematical structures of classical equilibrium statistical mechanics; With a foreword by Giovanni Gallavotti and Gian-Carlo Rota. MR511655 (80g:82017)

[14] D. Ruelle, Generalized zeta-functions for Axiom A basic sets, Bull. Amer. Math. Soc. 82 (1976), no. 1, 153-156. MR0400311 (53 \#4146)

[15] David Ruelle, Repellers for real analytic maps, Ergodic Theory Dynamical Systems 2 (1982), no. 1, 99-107. MR684247 (84f:58095)

[16] A. Verjovsky and H. Wu, Hausdorff dimension of Julia sets of complex Hénon mappings, Ergodic Theory Dynam. Systems 16 (1996), no. 4, 849-861, DOI 10.1017/S0143385700009147. MR 1406438 (97g:58143)

[17] Christian Wolf, Dimension of Julia sets of polynomial automorphisms of $\mathbf{C}^{2}$, Michigan Math. J. 47 (2000), no. 3, 585-600, DOI 10.1307/mmj/1030132596. MR.1813546 (2002a:37072)

[18] Lai Sang Young, Dimension, entropy and Lyapunov exponents, Ergodic Theory Dynamical Systems 2 (1982), no. 1, 109-124. MR684248(84h:58087)

Mathematics Institute, University of Warwick, Coventry, CV4 7AL, United Kingdom E-mail address: mpollic@maths.warwick.ac.uk 